vol. $27-n^{\circ} 1 \mid 2011$

La construction de l'altérité dans l'espace noir atlantique : Etats-Unis - France - Caraïbes - Amérique latine

\title{
Negritos et mestizos à Mérida dans la première moitié du XXe siècle. Métissage, région, race
}

Negritos and Mestizos in Merida at the Beginning of the 20th. Mestizaje,

Region, Race

Negritos y mestizos en Mérida en la primera mitad del siglo XX. Mestizaje, región y raza

\section{Elisabeth Cunin}

\section{Q OpenEdition}

Journals

\section{Édition électronique}

URL : https://journals.openedition.org/remi/5393

DOI : $10.4000 /$ remi.5393

ISSN : $1777-5418$

Éditeur

Université de Poitiers

Édition imprimée

Date de publication : 1 juin 2011

Pagination : 147-169

ISBN : 979-10-90426-00-9

ISSN : 0765-0752

Référence électronique

Elisabeth Cunin, « Negritos et mestizos à Mérida dans la première moitié du XXe siècle. Métissage, région, race », Revue européenne des migrations internationales [En ligne], vol. 27 - $n^{\circ} 1$ | 2011, mis en ligne le 01 juin 2014, consulté le 14 avril 2022. URL : http://journals.openedition.org/remi/5393 ; DOI : https://doi.org/10.4000/remi.5393 


\title{
Negritos et mestizos à Mérida dans la première moitié du XXe siècle. Métissage, région, race
}

\author{
Elisabeth CUNIN ${ }^{1}$
}

\begin{abstract}
A l'extrême sud-est du pays, entre Golfe du Mexique et Mer Caraïbe, la péninsule du Yucatán ${ }^{2}$ est connue, au Mexique, pour ses velléités autonomistes tant d'un point de vue politique que culturel (Careaga, 2000 ; Ramírez Carrillo, 2003 ; Taracena, 2007). La référence à une « identité yucatèque », s'appuyant sur une mise en valeur du passé maya et du métissage contemporain, marque un régionalisme omniprésent dans les discours quotidiens et institutionnels. Au début du XXe siècle, le contexte local est particulièrement agité (Orosa Díaz, 1988 ; Lapointe, 2006) : la Guerre des castes, qui a ensanglanté la péninsule pendant plus de cinquante ans, vient à peine de s'achever ; la révolution mexicaine s'étend au Yucatán et voit l'instauration de deux gouvernements socialistes, avec Salvador Alvarado et Felipe Carrillo Puerto ; la fin de la Première Guerre mondiale commence à marquer le déclin de l'industrie du henequén (sisal) et d'un système semi-esclavagiste fondé sur l'exploitation brutale de la population indienne ; les villes, en particulier Mérida, croissent de façon inédite. Il s'agit alors de faire entrer la péninsule, farouchement attachée à son autonomie et largement dominée démographiquement par la population indienne, dans la dynamique des politiques de métissage qui caractérisent l'après révolution de 1910 au Mexique (Florescano, 1997). L'indien, longtemps ignoré, méprisé et exploité, doit désormais être intégré dans ce Mexique révolutionnaire qui s'étend jusqu'à Mérida, mais aussi dans un Yucatán qui sort à peine d'un demi-siècle de Guerre des castes, conflit extrêmement complexe, qui n'en trouve pas moins une de ses
\end{abstract}

1 Chercheur IRD (Institut de Recherche pour le Développement) - CIESAS (Centro de Investigaciones y Estudios Superiores en Antropología Social, Mexique) - UQROO (Université du Quintana Roo, Mexique), Universidad de Quintana Roo, División de Ciencias Políticas y Humanidades, Edificio D, Boulevard Bahía s/n esq. Ignacio Comonfort, Col. del Bosque Chetumal, Quintana Roo, México C.P. 77019 ; elisabeth.cunin@ird.fr

2 Le Yucatán est à la fois le nom donné à l'ensemble de la péninsule et à un des États qui la compose, dont la capitale est Mérida. Les deux autres États de la péninsule, le Campeche et le Quintana Roo, sont des divisions nées de l'État du Yucatán. La référence à une « identité yucatèque » ou au « théâtre yucatèque » joue bien souvent sur cette ambiguïté (région et/ou État). 
origines dans la domination des populations indiennes par une petite partie de la population identifiée comme « caste divine ». L'époque est donc marquée par une tension particulière entre le national et le régional, entre modernité et tradition, entre homogénéité et hétérogénéité, entre indio et mestizo ${ }^{3}$.

La construction du métissage et l'intégration nationale ont été largement étudiées au Mexique (Florescano, 1997, 2002 ; Basave Benítez, 2002 [1992] ; Krauze, 2007) ; en cette année de bicentenaire de l'indépendance et de centenaire de la révolution, elles font l'objet de débats renouvelés ${ }^{4}$. Je propose ici d'étudier ces questions avec un regard différent en m'intéressant à un acteur exclu tant du métissage que de la nation : le noir, qu'il soit défini en termes phénotypiques, biologiques, culturels, d'origine, etc. Le programme intitulé la " troisième racine » a certes contribué à réintroduire le noir dans les récits sur le métissage et la nation, mais sans véritablement les mettre en cause (Hoffmann, 2006) : il s'agissait d'ajouter un troisième terme au binôme indien-européen, qui venait donc enrichir le métissage national, mais n'interrogeait pas la logique socio-historique du métissage, ni son association à la construction nationale.

L’appréhension de la région du Yucatán est marquée par un biais idéologique, politique et épistémologique qui empêche de donner aux descendants d'africains la place qu'ils ont occupée dans la formation de la société locale. En particulier, le petit nombre de travaux universitaires sur les populations noires du Yucatán ${ }^{5}$ renvoie certes en premier lieu à la faiblesse et à la dispersion des sources, au moindre rôle joué par l'économie de plantation sucrière, à la mise en esclavage des populations indiennes plus que des populations noires, à l'importance du commerce illégal d'esclaves, etc. Mais il me semble qu'il révèle aussi une certaine représentation implicite du noir qui n'est considéré ni comme un acteur de l'histoire, ni comme une expression d'altérité, et renvoie à une approche méthodologique qui interdit de penser la différence autrement que sur le modèle indien d'ethnicité, basé sur la référence au territoire, à la langue, à l'identité. Je me propose ici de contribuer à une autre interprétation de cette présence noire dans le Yucatán du XXe siècle et de participer ainsi aux réflexions sur le processus même du métissage, en faisant l'hypothèse que le noir en fut un acteur central, tout en disparaissant lui-même de/dans ce métissage. Il ne s'agit donc pas tant de dénoncer ou de réparer l' « oubli » des populations noires, en réintroduisant, notamment, le noir dans le métissage et dans la nation, sous la forme d'une « troisième racine », que d'interroger le statut du noir au début du XXe siècle pour mieux comprendre l'acceptation ambiguë d'une politique du métissage dans le Yucatán.

Pour cela, je m'intéresserai tout d'abord à un des symboles incontestés du Yucatán, et même de l'« identité yucatèque » dans son double rapport à l'indien et à l'eu-

3 J'utiliserai les termes « noir », « mestizo », « mulâtre », « indien » sans majuscule, afin de renvoyer à une catégorie d'appartenance socialement construite, et au singulier, dans une approche idéaltypique wébérienne. La majuscule sera conservée dans les références où elle est employée.

4 Voir par exemple le site officiel http://www.bicentenario.gob.mx/

5 Qui contraste avec l'omniprésence de la « question maya ». Voir néanmoins, entre autres : Aguirre Beltrán, 1989 (1946) ; Redondo Domínguez, 1994 ; Fernández Repetto et Negroe Sierra, 1995 ; Zabala, Cucina, Tiesler, Neff, 2004 ; Campos García, 2005 ; Collí Collí, 2005 ; Restall, 2005 ; Victoria Ojeda, Canto Alcocer, 2006. 
ropéen, le teatro regional yucateco, aujourd'hui encore associé aux générations successives de la dynastie Herrera, à l'actrice Ofelia Zapata "Petrona » dans son rôle de la mestiza, au feuilleton télévisé de la famille maya Chulim, autant d'acteurs qui ont fait le succès de ce théâtre populaire. De fait, le théâtre régional naît, au début du XXe siècle, en même temps que plusieurs expressions artistiques qui visent à représenter le " métissage à la yucatèque » et dont la guaracha, genre musical d'origine cubaine, La Mestiza de Cirilo Baqueiro, ou le roman La Mestiza d'Eligio Ancona Castillo sont les précurseurs. Spectacle de divertissement populaire, le théâtre yucatèque repose sur une caricature de la société et met en scène des personnages récurrents, que le public est censé connaître et reconnaître d'une pièce à l'autre. De fait, à côté du mestizo et de la mestiza, du propriétaire blanc ou du travailleur indien, on trouve également le chino (incarné notamment par l'acteur Daniel Herrera, de la dynastie d'acteurs et de compositeurs du même nom) ou le turco $^{6}$, qui symbolisent les migrants économiques arrivés dès la fin du XIXe siècle dans la péninsule. Mais également le negrito, présent dans la première moitié du siècle avant de disparaître complètement. Il ne s'agit pas d'acteurs noirs (ou chinois, ou syro-libanais ou même mestizos ou indiens), mais d'acteurs blancs jouant les noirs, les chinois ou les « turcs ».

Le travestissement blanc/noir renvoie à un phénomène bien connu dans les Amériques et la Caraïbe : celui du blackface présent aux États-Unis dès le début du XIXe siècle, mais aussi dans le théâtre bufo cubain avec le personnage du negrito, ainsi que dans d'autres théâtres nationaux ${ }^{7}$ et carnavals - la guaranducha de Cozumel (Ramírez Canul, 2001) ou le carnaval de Mérida (Irigoyen Rosada, 1976) pour rester dans la péninsule. Le blackface est un " spectacle du même et de la différence » (Lhamon, 2008 : 212) qui met en scène des acteurs blancs dont le visage est peint en noir. Il se rit du noir bien sûr et constitue à ce titre une forme de racisme ; mais il signifie aussi l'appropriation des gestes et des musiques des noirs par des acteurs et des spectateurs blancs ou mestizos et, au-delà, leur adhésion ambiguë à une certaine représentation de la culture noire.

Dans une première partie, l'analyse du théâtre yucatèque me donne l'occasion de sortir de la confrontation indien/blanc, péninsule/centre pour situer mes propos dans un cadre d'analyse plus large, qui renvoie à une multiplicité d'appartenances (negrito, chino, turco) et qui inscrit le Yucatán dans l'espace Caraïbe autant que dans l'espace national.

Or le noir n'est pas seulement un personnage de théâtre (ou de carnaval) : il est également un acteur de la vie quotidienne de Mérida dans la première moitié du XXe siècle. Absent du récit historique républicain qui se réfère désormais à une citoyenneté indifférenciée, absent des travaux anthropologiques car seul l'indien incarne l'altérité, le noir est pourtant présent dans la vie ordinaire. Il est donc nécessaire de varier les sources pour retrouver sa trace aux XIXe et XXe siècles : c'est pourquoi je m'intéresserai, dans une

6 Nom donné aux migrants syro-libanais arrivés en Amérique latine dès le XIXe siècle. Sur les libanais dans le Yucatán, voir Cuevas Seba, Mañana Placensio, 1990. Sur les chinois, Cervera, 2007.

7 Sur le blackface, voir en particulier : pour les États-Unis, Lott, 1993 ; Béthune, 2007 ; Lhamon, 2008 ; pour Cuba et le teatro bufo : Leal, 1975 et 1980 ; Frederick, 2001 ; Lane, 2005 ; Martiatu, 2009; sur l'adaptation du blackface à Puerto Rico : Rivero et Rivero, 2004 ; au Pérou : Feldman, 2009 ; en Afrique : Cole, 1996 ; dans la Caraïbe : Suárez Durán, 2008. 
deuxième partie, aux récits sociaux sur Mérida dans la première moitié du XXe siècle, récits dans lesquels le noir apparait comme un des personnages populaires de la ville. Entre indifférence à la différence et différence non significative, j'analyserai le statut du noir, à la fois racialisé, exclu et familier.

Enfin, je reviendrai sur le métissage appréhendé à partir de la place du noir dans la société yucatèque de la première moitié du XXe siècle. Si le blackface a été associé à une forme de transgression sociale aux États-Unis (Lhamon, 2008) et à la construction de la nation à Cuba (Lane, 2005), j'interrogerai son rôle dans le Yucatán, notamment dans les politiques du métissage de la première moitié du XXe siècle.

Cet article se situe donc au carrefour d'une approche culturelle, historique et anthropologique, et s'appuie sur la révision de sources qui ne sont généralement pas explicitement consacrées aux populations noires ou par rapport auxquelles la catégorie «noir » ne constitue pas un objet d'étude : archives du Centro de Investigaciones Escénicas de Yucatán (coupures de presse, pièces de théâtre), principal quotidien de la péninsule (Revista de Yucatán qui devient Diario de Yucatán à partir de 1925), ouvrages décrivant la vie quotidienne à Mérida dans la première moitié du XXe siècle, ouvrages portant sur le théâtre régional yucatèque, photothèque de l'Universidad Autónoma de Yucatán.

\section{DU YUCATÁN À LA CARAÏBE : SORTIR DU FACE À FACE INDIEN/BLANC}

Le théâtre régional yucatèque est intrinsèquement lié aux bouleversements politiques, sociaux et économiques qui marquent le début du XXe siècle au Mexique ; il est l'enfant de la révolution mexicaine et, au niveau plus local, des transformations introduites par Salvador Alvarado et Felipe Carrillo Puerto (Echánove Trujillo, 1944 : 774). De fait, les profonds changements sociaux-économiques de l'époque se reflètent dans la démocratisation de l'accès au théâtre. Dans la ville de Mérida, capitale régionale aux prétentions culturelles, à côté du classique et imposant Théâtre Péon Contreras, qui continue à présenter des œuvres européennes ou inspirées de la littérature européenne, apparaissent à la fin du XIXe et au début du XXe siècle le Circo Teatro, les théâtres Mérida, Olimpia, Apolo, Colonial, qui seront directement associés au succès du teatro regional yucateco.

Le discours savant sur le théâtre régional yucatèque l'inscrit dans un double héritage, espagnol et indien ; pourtant ce théâtre, comme de nombreuses autres expressions culturelles de l'époque, doit également être replacé dans le contexte des circulations caribéennes qui caractérisent la région et, notamment, les échanges entre le Yucatán et Cuba ${ }^{8}$. Si le théâtre yucatèque célèbre une identité régionale, qui serait issue de la rencontre entre populations européenne et indienne, il n'en met pas moins en scène une diversité beaucoup plus large. Il faut en effet rappeler que le théâtre régional yucatèque n'est pas réductible aux seuls personnages du mestizo et de la mestiza, de l'indien (le vinic, la xunán, etc.) ou

8 La péninsule du Yucatán a jusqu'alors plus de contacts avec la Caraïbe qu'avec le reste du Mexique et son ancrage caribéen ne se limite pas à Cuba : il s'étend aux États-Unis (Nouvelle-Orléans, Texas), à l'Amérique centrale, notamment le Belize, à la Colombie. 
du blanc (propriétaire d'hacienda et sa famille, administrateur) : il donne à voir la diversité qui caractérise Mérida et le Yucatán au début du XXe siècle. Et cette diversité, au-delà de la dimension nationale, renvoie à l'appartenance caribéenne de la péninsule.

\section{Le récit officiel : espagnols, mayas et identité régionale}

En 1947, apparaît un ouvrage de Cervera Andrade sur le théâtre régional yucatèque. Ce livre naît du souhait de Leopoldo Peniche Vallado, Directeur Général des Beaux-arts à Mérida, d'éditer les textes de théâtre régional, généralement dispersés et non diffusés. Cet ouvrage met en lumière une double généalogie du théâtre, européenne et indienne : il rappelle l'« influence logique », qui inscrit le théâtre dans une « affinité envers tout ce qui est espagnol » (Cervera Andrade, $1947: 23$ ); et, dans le même temps, précise que le théâtre tire " son origine des cérémonies traditionnelles des indiens de l'époque coloniale » (Cervera Andrade, 1947 : 24). Les rituels sacrés, les croyances religieuses, les fêtes populaires mayas sont alors longuement évoqués. De son côté, la très institutionnelle Enciclopedia Yucatanense insiste davantage sur le passé européen du théâtre régional. Le chapitre 11 qui lui est entièrement consacré (Echánove Trujillo, 1944) fait remonter les ancêtres du teatro regional yucateco à la fin XIXe siècle avec de « timides adaptations » des zarzuelas ${ }^{9}$ espagnoles au contexte yucatèque. Dans la même encyclopédie, Gamboa Garibaldi (1946) rappelle que le XIXe siècle fut avant tout celui des classiques et romantiques européens : les auteurs et les acteurs sont italiens, français, espagnols, avec néanmoins une nette préférence pour la zarzuela espagnole. Petit à petit, ces œuvres venues d'Europe se combinent avec celles des auteurs locaux comme Wenceslao Alpuche ou Mariano Trujillo.

Plus récemment, dans un ouvrage de 1987, on retrouve l'idée d'un ancrage dans un passé indien ancestral : la tradition théâtrale yucatèque vient « de l'époque précolombienne » (Muñoz, 1987 : 10). Cette analyse fait l'objet du premier chapitre : « Théâtre Maya Précolombien ». Puis sont évoqués les effets de la colonisation, marquée par la domination de la culture européenne avec le «Théâtre de l'évangélisation » (chapitre 2). Le théâtre du XIXe siècle est caractérisé par l'influence européenne, en particulier espagnole (chapitre 3). Enfin, le dernier chapitre présente le théâtre contemporain, en insistant sur son originalité : son caractère régional, résultat de cette rencontre entre mondes européen et maya. Ce théâtre reflète « notre manière d'être et de sentir, avec notre langage quotidien et notre sens de l'humour si yucatèque qui continue d'être aujourd'hui un "cas" à part dans la République mexicaine et peut-être sur le continent américain » (Muñoz, 1987 : 88). Car, avant tout, le teatro regional est présenté comme «l'esprit de la population yucatèque » (Cervera Andrade, 1947 : 14), né dans un de ces lieux où " palpite le cœur yucatèque " (Cervera Andrade, 1947 : 18). Les scènes représentent ainsi l' " esprit » de la région, les légendes et coutumes locales (Cervera Andrade, 1947 : 22). Le langage est une illustration de cette fusion entre cultures européenne et indienne, qui serait typique du Yucatán et que ne manquent pas de mentionner les exégètes du théâtre régional, avec un espagnol mâtiné de mots, d'expressions et de tonalités mayas (Echánove Trujillo, 1946 : 287).

9 Genre musical théâtralisé apparu en Espagne au XVIIe siècle. 


\section{Les échanges oubliés avec Cuba : le Yucatán au cour des circulations caribéennes}

Cervera Andrade se réfère aux théâtres italien, français, espagnol et même au théâtre argentin. Mais jamais au théâtre cubain. De même Muñoz fait quelques rapides références au théâtre bufo en informant que des troupes cubaines fréquentaient la péninsule et en lui consacrant une page (Muñoz, 1987 : 74-75). Pourtant, nombreux sont les exemples qui nous montrent l'importance des échanges entre théâtres yucatèque et cubain ${ }^{10}$ : dès 1831, le directeur d'un théâtre de Mérida écrit à La Havane pour confirmer la venue d'une troupe qui doit inaugurer son théâtre (Gamboa Garibaldi, 1946 : 122). En 1845, M. Méndez Sierra, entrepreneur de Mérida, se rend à La Havane et revient avec plusieurs acteurs et actrices cubains (Registro Yucateco, 1845 : 193). En 1868, Enrique Guerrero, est un des " plus importants entrepreneurs et organisateurs de compagnies de théâtre bufo cubain venu au Mexique », en particulier à Veracruz et à Mérida (Montenegro Rolon, del Valle Arroyo, s.d. : 81). En 1919, le Teatro Olimpo fait venir « la Chelita Criolla » de La Havane alors que le cubain Arquimedes Pous triomphe déjà dans ce même théâtre. En 1926, Héctor Herrera, un des « pères fondateurs » du teatro regional yucateco, fait une tournée à La Havane. En 1935, la compagnie cubaine Camelia joue au Théâtre Péon Contreras, le plus prestigieux de Mérida. On pourrait ainsi multiplier les exemples qui montrent que, contrairement à ce que laisse entendre (par omission) le « récit officiel », Cuba a joué un rôle fondamental dans la naissance du théâtre régional yucatèque (tout comme, inversement, les artistes yucatèques ont certainement eu une influence sur la scène théâtrale et musicale cubaine). Juan Manzanilla Dorantes (1994), dans un article publié à Mérida, est l'un des rares analystes à s'intéresser directement aux liens entre le théâtre bufo cubain et le théâtre régional yucatèque ${ }^{11}$. Selon lui, ce n'est qu'après son voyage à La Havane dans les années 1920 qu'Héctor Herrera va véritablement commencer à développer le théâtre régional, qui vient ainsi remplacer les opérettes et les zarzuelas qui dominaient encore la scène de Mérida.

\section{Le personnage du negrito}

Le travail de J. Manzanilla est également un des rares à évoquer la présence, largement passée sous silence ailleurs, du personnage du negrito dans le théâtre yucatèque (voir photographies 1 et 2). De fait les textes consacrés au théâtre régional soit « oublient» de faire référence au fait que les acteurs Pous, Arredondo, Camejo ou Rogelini jouaient un noir, soit mentionnent le personnage du negrito au détour d'une phrase, sans juger utile de lui accorder un quelconque commentaire. Dans un cas comme dans l'autre, le noir est « non signifiant », il n'est pas socialement pertinent en tant qu' « autre ». Pourtant, il est déjà présent dans les pastorales de l'époque coloniale, considérées elles aussi comme

10 Échanges qui s'inscrivent eux-mêmes dans des circulations plus larges, au niveau économique, politique, culturel, migratoire (Maldonado, 1988 ; Bojorquez, 1986 et 2000 ; Ramos Smith, 1991 ; Novelo, 2009 ; Perez Montfort, 2007 ; Ávila Domínguez, Pérez Montfort, Rinaudo, à paraître).

11 Ce travail s'inscrit dans le cadre d'un projet plus large portant sur la dynamique Yucatán-Cuba coordonné par Victoria Novelo au CIESAS Peninsular. Voir aussi Antonio Prieto Stambaugh et Oscar Armand Garcia, 2007 ; Tuyub, 2005 : 17-18. 


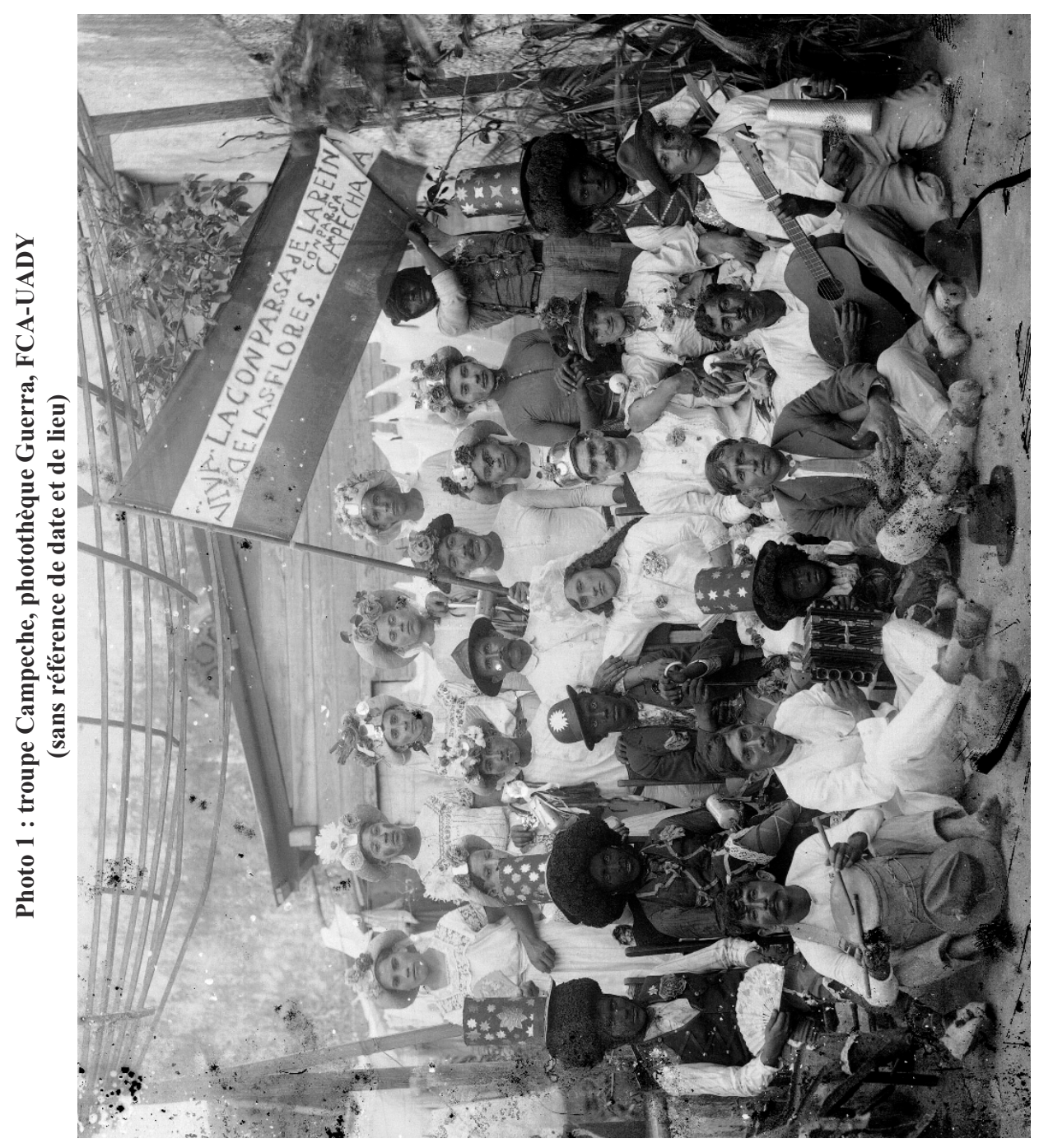


Photo 2 : théâtre Duarte, photothèque Guerra, FCA-UADY

(sans référence de date et de lieu)

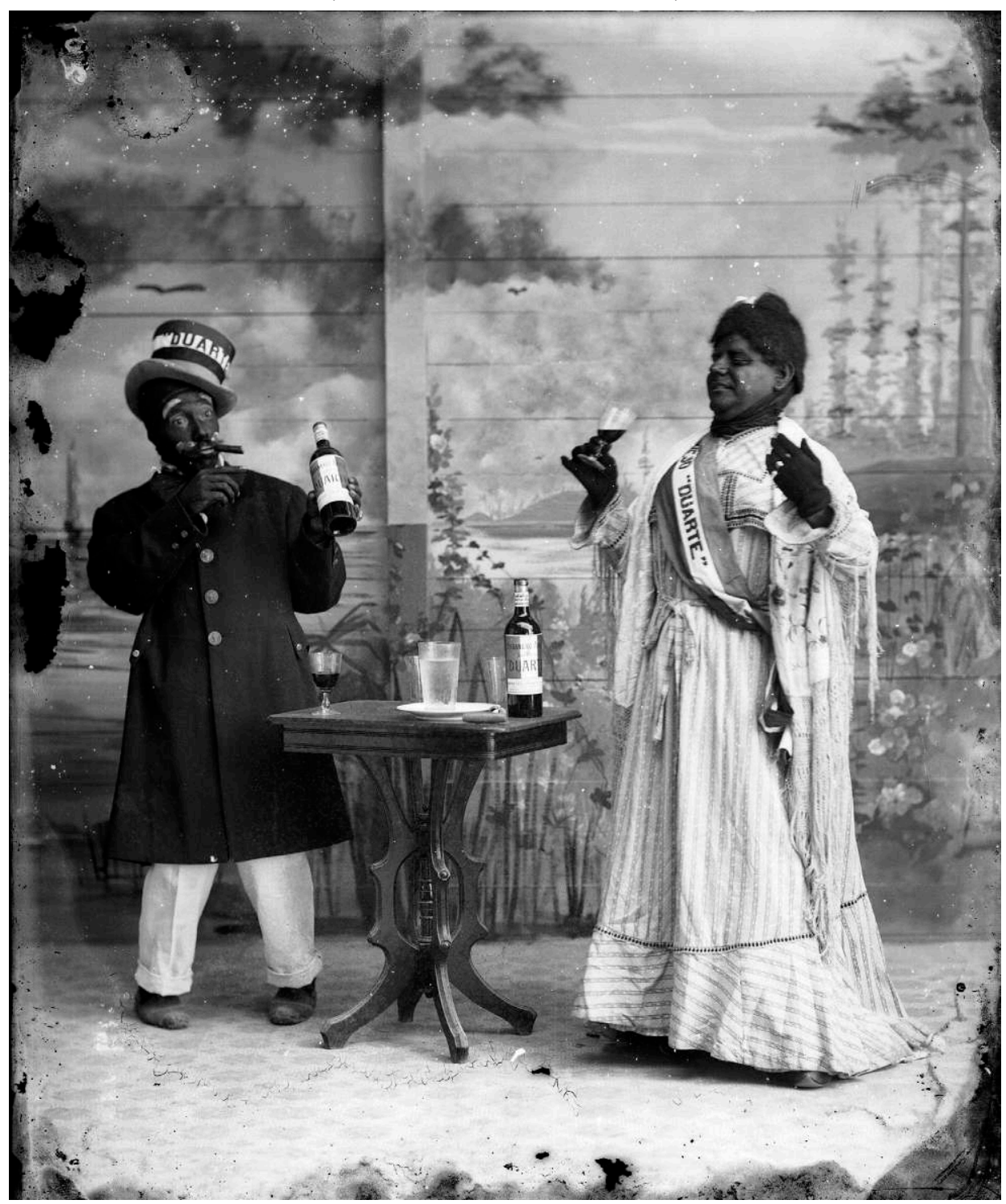


un des ancêtres du théâtre régional yucatèque (Tuyub, $2005: 11$ ) qui mettent en scène les personnages du Negro et de la Negra. De même une des œuvres annonciatrices de l'ancrage régional du théâtre yucatèque est Diego el mulato, écrite en 1846 par José Antonio Cisneros et dont le personnage principal est celui de Diego, un pirate mulâtre.

Surtout, plusieurs acteurs cubains ont incarné avec succès le personnage du negrito à Mérida et dans le Yucatán ${ }^{12}$ : Arquimedes Pous, Alfonso Rogelini, Arnaldo Camejo, Enrique Arredondo (voir photographie 3). Ces acteurs jouent un répertoire cubain, mais aussi des œuvres directement inspirées du Mexique : Mérida Carnaval, Yucatán Souvenir, De México Vengo, écrites et composées sur place, avec des personnages et des événements renvoyant à la société de Mérida. D'autres pièces sont plus directement dédiées aux populations noires et mulâtres : Las mulatas de Bam-Bay, El brillante Negro par exemple. Je n'ai malheureusement pas pu retrouver les textes de ces pièces (beaucoup d'entre elles étaient écrites à la veille de la représentation et n'étaient pas éditées ni conservées) et il n'est donc pas possible de savoir si elles traitent du Yucatán ou de Cuba. Mais l'affichage du titre, la participation d'acteurs jouant le personnage du negrito permettent néanmoins de confirmer l'importance de la mise en scène du " noir », à côté de celle du métis, de l'indien, du blanc, du chinois et du « turc ».

Les annonces de spectacles publiées dans la Revista de Yucatán puis dans le Diario de Yucatán permettent de suivre la présence de ces acteurs à Mérida au début du XXe siècle. Je ne retiendrai qu'un exemple, celui d'Enrique Arredondo, qui occupe la scène yucatèque durant les mois de novembre et décembre 1943, avec un grand nombre de pièces. Le Diario de Yucatán le présente à plusieurs reprises comme « le negrito Arredondo », « le génial negrito » et fait référence aux pièces dans lesquelles il joue le rôle du negrito : Un negrito en Yucatán, Los Negros del Manglar, Tumbando caña avec ses negritos rumberos, El Negro que tenía alma blanca, etc. Il participe notamment au grand succès Tunkules y maracas $^{13}$ présenté sur les affiches comme «Une revue avec une âme yucatèque et une saveur cubaine ». La pièce est écrite par Fernando Mediz Bolio, dirigée par E. Arredondo et jouée par les acteurs les plus connus de l'époque, Héctor Herrera et Ofelia Zapata. Elle est le symbole de la rencontre « entre les deux théâtres, entre deux des plus importantes traditions scéniques de la Grande Caraïbe » (Prieto Stambaugh et Armand García, 2007) et sera d'ailleurs suivie par une tournée à Cuba des artistes yucatèques en $1945^{14}$. Tunkules y maracas semble avoir marqué l'année : les annonces du Diario de Yucatán parlent ainsi de « grandiose présentation populaire » ( $1^{\text {er }}$ novembre 1943), d'un « public [qui] ovationne nuit après nuit » $(9$ novembre 1943), de " première sensationnelle de la merveilleuse revue cubano-yucatèque » (3 décembre 1943), de " succès théâtral de l'année à Mérida » (8 décembre 1943). La pièce est jouée pratiquement tous les jours des mois de novembre

12 Le rôle du negrito semble avoir été réservé à des acteurs blancs cubains (à l'inverse du chino ou du turco, joués par des acteurs blancs mexicains) à l'exception d'Arnaldo Camejo. Gilma Tuyub mentionne la venue d'une compagnie de bufo cubain dès la fin du XIXe siècle à Mexico puis à Mérida (Tuyub, 2005 : 17-18) avec des pièces comme Los negritos catedráticos et La duquesa de Haití et des personnages de mulata et de negro.

13 Du nom de deux instruments de musique, l'un yucatèque, l'autre cubain.

14 Ces années signent l'apogée du théâtre régional yucatèque avec la naissance de la famille Chulim, une tournée dans la ville de Mexico et les premières apparitions à la télévision. 
et décembre, alors que les pièces ne restent généralement que quelques jours à l'affiche. Le départ pour Cuba d'Enrique Arredondo sera l'occasion de nombreuses festivités dont les noms des artistes invités sont évocateurs (Los Negros del manglar, El Negro Pachuco, los Diamantes negros) et d'un concours de danzón et de rumba. Le Diario de Yucatán évoque ainsi une « grandiose présentation extraordinaire en l'honneur du premier acteur et directeur, le sympathique negrito Enrique Arredondo » (23 décembre 1943). Dans le même temps, Daniel Herrera triomphe lui aussi au Circo Teatro dans son rôle de chino avec des œuvres qui sont elles-mêmes qualifiées de «bufonadas asiatiques » et des pièces comme La coleta de Fu-Man-Chu, El general Chan-Chan-Seck, Quiereme Koy-Koy (photographie 4). Le 23 décembre, le chino Herrera suspend sa fonction et participe à l'hommage rendu à Enrique Arredondo ; puis le 24, c'est l'inverse, Enrique Arredondo assiste à l'hommage rendu à Daniel Herrera. Une pièce spéciale est écrite à l'occasion du départ d'Enrique Arredondo : A La Habana me voy. Et, pour la première fois, le negrito dévoile en public sa métamorphose : « venez voir comment se transforme Arredondo de blanc à noir. Pour la seule et unique fois, il se peindra le visage devant le public ».

On voit ainsi que le « noir » est « oublié », non signifiant dans les ouvrages traitant du théâtre régional yucatèque ; pourtant les archives de presse et photographiques nous montrent qu'il fut bel et bien un des acteurs de ce théâtre, au moins dans la première moitié du XXe siècle. En outre, il incarne l'étranger et, plus précisément, le cubain : les acteurs, les auteurs et même bien souvent les musiciens ou une partie de la troupe des spectacles dans lesquels joue le negrito sont originaires de Cuba. Alors que le mestizo, l'indien, le blanc, le chinois ou le « turc » sont joués par des acteurs mexicains, le rôle du negrito semble être exclusivement réservé aux acteurs cubains, l'associant ainsi à l'éloignement, à l'étrangeté, voire à l'exotisme. Cette représentation du « noir » est-elle limitée à l'espace théâtral et, au-delà, au champ culturel, ou se retrouve-t-elle dans des pratiques sociales plus ordinaires?

\section{LE STATUT DU NOIR À MÉRIDA}

La quasi absence de référence au negrito dans les écrits sur le théâtre yucatèque et la disparition des œuvres de l'époque rendent difficile le travail sur ce personnage. Néanmoins, on dispose d'une autre source tout à fait utile : les récits sociaux sur Mérida au début du XXe siècle. De fait, il faut rappeler que le théâtre régional vise avant tout à représenter des personnages populaires, issus de la vie quotidienne, des « personnages types extraits des rues et des places de Mérida et de ses environs » (Prieto Stambaugh et Armand García, 2007). Montejo Baqueiro voit dans le negrito une imitation des noirs de la vie quotidienne (1986 : 270). Gilma Tuyub (2005: 55) rappelle elle aussi qu'Arnaldo Camejo «s'est distingué en imitant le Noir Miguel, un vendeur de glaces, qui fut très connu dans la première moitié du XXe siècle en raison de ses chansons singulières ».

Au début du siècle, à Mérida, le noir est, comme on va le voir, un personnage populaire. Alors que la mémoire officielle ne retient de la première moitié du XXe siècle que les auteurs de romans, de poésie, de théâtre, célébrant la civilisation maya, il existe aussi une littérature plus sociale, s'adressant plus directement à une population urbaine en pleine explosion. Parmi les personnages typiques qui fréquentent les rues de Mérida, on 
Photo 3 : Diario de Yucatán, 19 mars 1942

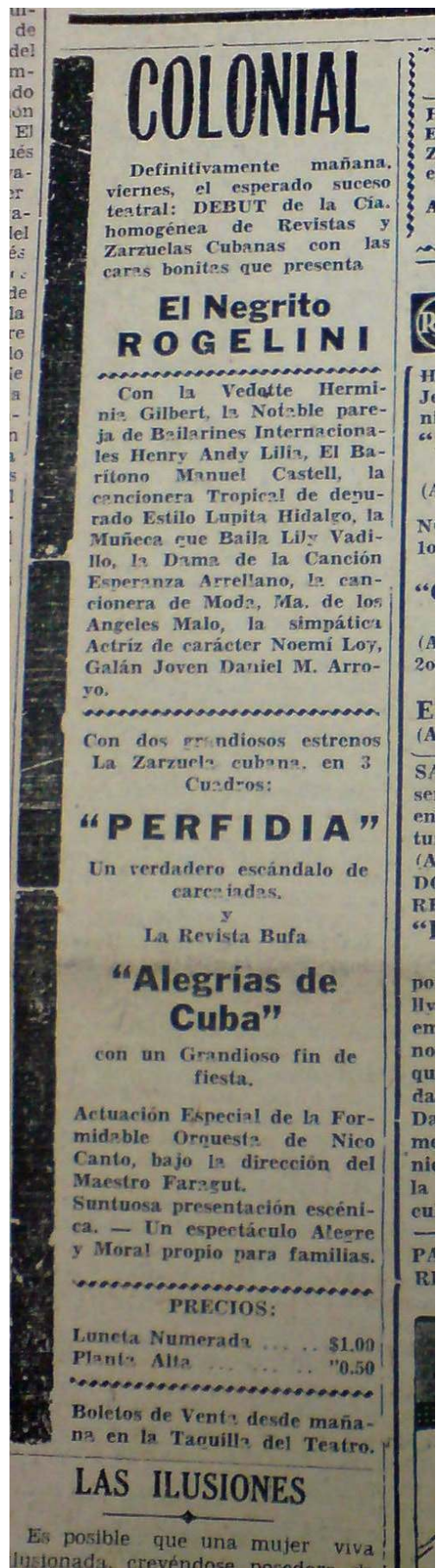


Photo 4 : Diario de Yucatán, 23 décembre 1943

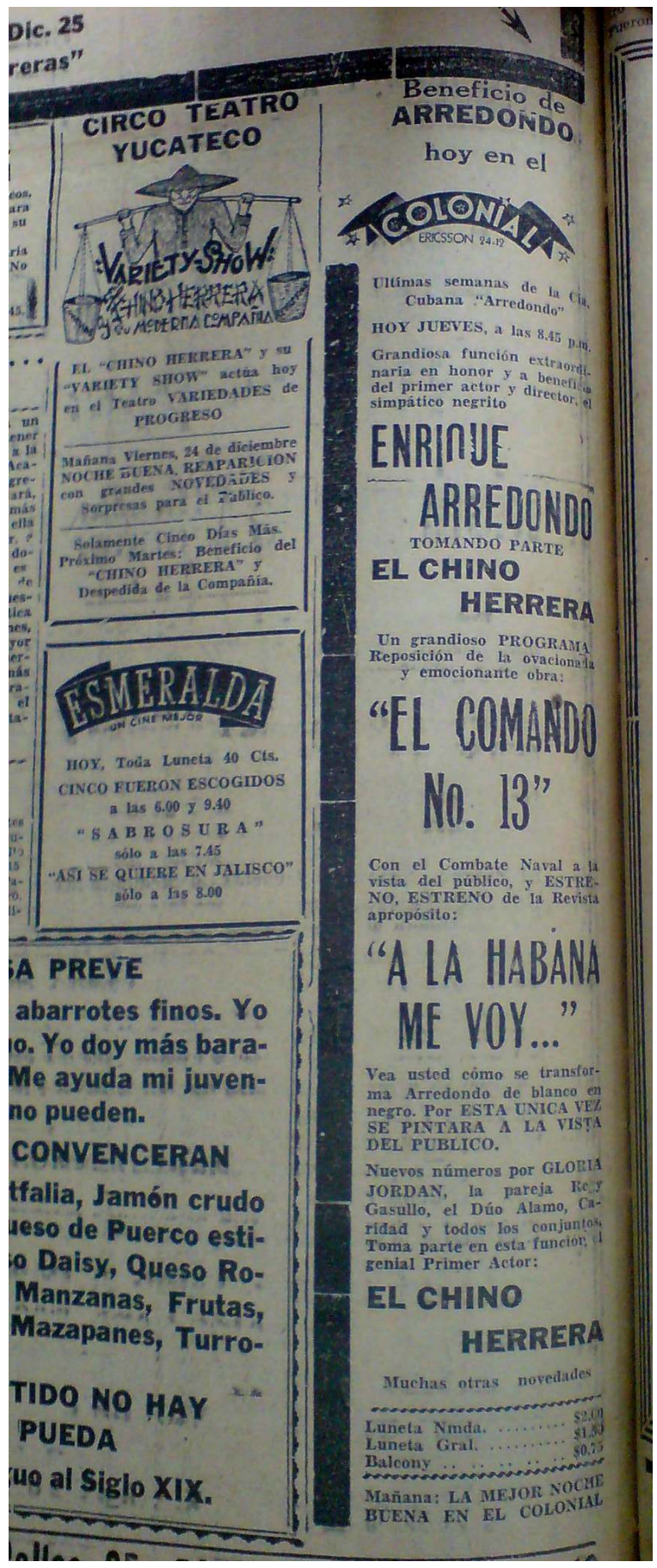


trouve plusieurs noirs et, en tout cas, bien plus de noirs que d'indiens. Dans sa description de Mérida dans les années 1920 (Mérida en los años veinte), entre travail historique et chronique de la vie quotidienne, Francisco Montejo Baqueiro retient trois personnages noirs : le Negro Timbilla, le Negro Miguel et le Negro Crispín. Le Negro Timbilla (Montejo Baqueiro, $1986: 267-270$ ) est « noir de race et originaire de Cuba ». Cireur de chaussures, il occupe des espaces particulièrement fréquentés et symboliques de la ville (Palacio de Gobierno, glacier Colón). Il souffre d'une infirmité congénitale aux jambes qui l'empêche de marcher normalement et est ainsi repérable à sa canne. S'ajoutant à la maladie, sa couleur apparaît également comme un handicap incontournable : « le noir de sa peau et de son corps déformé était contrebalancé par la grâce naturelle de tous les cubains ». Pour achever ce portrait peu élogieux, Montejo Baqueiro rappelle que Timbilla est vulgaire (« de sa bouche aux grosses lèvres sortaient des blasphèmes terribles ») et boit excessivement dans les cantinas qu'il fréquente assidûment. Car en plus de ses tares physiques, Timbilla est incapable de vivre en société : « le Negrito était rancunier et anticonformiste, et même en rébellion ouverte contre son sort ». Lors d'une manifestation de travailleurs sur la place centrale de Mérida en 1915, protestant contre « l'oppression, le capitalisme, les riches », le Negro Timbilla participa aux échauffourées et à l'invasion de la Cathédrale dans laquelle il dansa sur la statue du Christ, témoignage de son « ressentiment caché et de son complexe d'infériorité ». Pourtant, l'auteur répète aussi à plusieurs reprises que Timbilla fut « très connu et populaire », « ami de tous » et qu'il jouissait d'une « popularité de rue ».

El Negro Miguel (Montejo Baqueiro, 1986 : 270-273 ; Muñoz, 1987 : 226), " couleur acajou », est quant à lui vendeur de glaces « avec sa caisse de cônes glacés sur la tête et son refrain sympathique devenu chanson : glaces, glaces, glaces, glaces, glaces, elles sont à l'ananas, à la noix de coco et au caramel ». Dans son cas, personne ne sait vraiment comment il est arrivé à Mérida, mais pour Montejo Baqueiro il ne peut qu'être cubain, au point de se référer à l'histoire des familles riches de Mérida qui voyageaient à Cuba à la fin du XIXe et qui ne manquaient pas de visiter « un Hospice, sous la responsabilité de religieuses, Sœurs de la Charité, en face du Parc Antonio Maceo ». Il n'était pas rare que ces familles reviennent à Mérida avec un serviteur noir ${ }^{15}$ et Miguel pourrait être l'un d'eux, même si l'auteur reconnaît que cette version n'est pas confirmée. Tout comme Timbilla, il n'a pas été épargné par la nature : victime d'une épidémie de « variole noire ", il fut hospitalisé mais conserva certaines déformations sur le visage et perdit un œil. Il n'échappe pas au regard stéréotypé de son époque qui en fait un personnage « de caractère jovial et sympathique » et un « singe savant» dans les corridas du Circo Teatro. Lui aussi est connu par plusieurs générations d’habitants de Mérida (« un des personnages les plus connus et populaires de cette époque. Tout Mérida le connaissait, tout le monde lui parlait ») et sa chanson était « connue dans tous les coins de la ville et reprise par les habitants et les visiteurs ». Il est d'ailleurs tellement populaire qu'il a posé pour l'artiste yucatèque Juan Gamboa Guzman et inspiré un danzón à D. Ernesto Mangas intitulé El negro Miguel, qui sera diffusé avec succès sous forme de disque et présenté lors du carnaval de 1919 (Montejo Baqueiro, $1986: 276)^{16}$.

15 Le récit fait d'ailleurs apparaître un nouveau personnage-type noir, Roberto, conducteur de calèches.

16 Montenegro Rolon et del Valle Arroyo (s.d. : 83) attribuent ce même danzón à Arquimedes Pous, acteur cubain qui interprétait le rôle du negrito. 
El Negro Crispín (Montejo Baqueiro, 1986 : 273-277), enfin, est venu de Cuba à la toute fin du XIXe siècle, même s'il est précisé plus loin que « nous ne savons pas comment il est venu ni qui l'a amené ». Encore plus que les autres, Crispín est marqué par la maladie - non véritablement précisée. Il se considérait comme un envoyé de Neptune, capable de prévoir la pluie. C'est ce qui le rendit populaire puisque tous les habitants de Mérida lui demandaient : " quand va-t-il pleuvoir ? »; ce qui lui permit de survivre en demandant l'aumône. Sa maladie le rendit donc sympathique avec une «popularité de rue qui en a fait, comme pour tant d'autres types populaires, un élément de la tradition de Mérida ». Lui aussi est décrit comme une personne a-sociale, voire un « paria du destin» : « dans sa misère et son imbécilité, Crispín vivait comme un grand seigneur à la marge de toute norme sociale », dormant sur les bancs publics. Finalement, " l'insignifiance de sa personne et peut-être la pigmentation de son épiderme n'ont pas contribué à sa vie en commun avec ses semblables ».

Au-delà de leurs singularités, on retrouve bien dans ces trois personnages des traits sociaux qui les apparentent à des types idéaux du noir au début du XXe siècle à Mérida : nécessairement cubain, même lorsqu'aucun élément empirique ne permet de l'affirmer ; a-normal tant du fait de sa composition biologique que de son statut social ; populaire, familier, quotidien au point de devenir un souvenir nostalgique de l'enfance ou un symbole de la ville.

Santiago Burgos Brito, dans son ouvrage Tipos pintorescos de Yucatán (Burgos Brito, 1946 : 175-185) retient lui aussi un personnage noir : «Macalú, le picador d'ébène ». On ne connaît pas l'origine de Macalú, mais on découvre les fiestas bravas du Circo Teatro auxquelles participait également El Negro Miguel et au sein desquelles Macalú est « le picador le plus populaire de Mérida ». Macalú est « noir très foncé, brillant, orgueilleux de sa race ». Comme chez Montejo Baqueiro, le noir est ici un personnage extrêmement populaire, « un des types populaires non seulement de Mérida, mais de tous les villages de l'État ». S. Burgos Brito insiste davantage sur les stéréotypes raciaux associés aux noirs : il est salué par des cris qui imitent ceux « de ses ancêtres de la jungle africaine », il est considéré comme un « être inférieur », condamné « à supporter les plaisanteries des petits et des grands ». On voit bien dans la description de Burgos Brito que le noir est avant tout un symbole plus qu'une personne, qu'il est à la fois familier et étranger, proche et distant. Il devient ainsi la menace que font planer les mères sur les enfants désobéissants : « les mères firent de Macalú le père Fouettard des enfants pas sages, le moyen le plus efficace de faire dormir ceux qui s'obstinaient à continuer leurs bêtises ». À la fois « anthropophage » et présent dans tous les foyers, « mauvais présage » et héros de spectacles populaires, Macalú est un personnage à la fois quotidien et anormal ${ }^{17}$, bien loin en tout cas des grands récits héroïques qui caractérisent les indiens et dont Canek serait, à la même époque, le symbole (voir Shrimpton, 2002, pour une approche littéraire ; Bracamonte y Sosa, 2004, pour une approche ethno-historique).

Le noir est à la fois exotique et quotidien, étranger et domestique. Montejo Baqueiro (1986 : 246) rapporte que, lors d'une fête de carnaval en février 1920, les enfants

17 Il faudrait aussi mentionner sa présence dans de nombreuses bombas yucatèques, petits poèmes populaires, parfois improvisés, échangés lors des fêtes. 
d'un collège choisirent différents déguisements : Louis XV, gitane, mexicaine, chinois, français, japonais, etc. Une jeune fille déguisée en noire, " ne trouvant personne pour danser avec elle à cause de sa "couleur", dansait seule en passant d'une pièce à l'autre avec un rythme cubain ». C'est le noir et pas l'indien qui fait sens en ville lorsqu'on veut se déguiser, changer de rôle. Et en même temps, le noir est problématique, dérange, plus que la gitane, le chinois ou la japonaise.

\section{LE NOIR, OXYMORE ET CATALYSEUR DU MÉTISSAGE}

Selon Lhamon, aux États-Unis, l'identification aux noirs a permis aux secteurs populaires de remettre en cause, le temps de la représentation théâtrale, les hiérarchies et les contrôles sociaux. En acclamant le personnage du noir, on affirme aussi une solidarité potentielle avec ceux qui incarnent la marginalité et la subalternité, tout en rejetant le paternalisme de la société dominante. Le noir sert de « miroir », « reflétant les codes du déracinement et de la transgression » (Lhamon, 2008 : 117) et permet ainsi aux classes populaires d'échapper au contrôle et à l'exploitation des élites et des classes moyennes. Jill Lane (2005), dans son étude du blackface à Cuba, s'interroge de son côté sur son rôle dans la construction d'une " identité cubaine » au moment où les guerres anticoloniales (entre 1868 et 1898) posent les premiers jalons de la nation cubaine. Les personnages du negrito et de la mulata ont légitimé l'opposition à l'Espagne, tout en garantissant la hiérarchie socio-raciale de la future nation.

Quel a été le rôle du blackface dans le Yucatán ? Il faut tout d'abord rappeler que le métissage, en dépit de la politique et de l'idéologie post-révolutionnaires, est loin d'être une évidence. Plusieurs pièces de Santiago Pacheco $\mathrm{Cruz}^{18}$ sont présentées dans les années 1920 à Mérida et dans le reste du Yucatán, avec en particulier la participation d'Héctor Herrera. Dans une logique post-révolutionnaire, elles dénoncent l'indien esclave, l'indien asservi, l'indien exploité et en appellent à son intégration au projet national. La Voz del amo (Pacheco Cruz, 1928) rappelle que seule la révolution a pu mettre un terme à l'esclavage dans la péninsule. Pourtant cette ambition politique et sociale n'est pas suffisante pour sauver les populations indiennes ; de fait, le métissage lui-même est la principale menace pour l'indien. L'œuvre de Santiago Pacheco Cruz témoigne de sa hantise du métissage, irrémédiablement vu comme une déchéance, une menace pour les populations indiennes. Entre l'indien millénaire et la disparition de l'indien, il n'y a aucune alternative possible. Pacheco Cruz semble surtout préoccupé par les conséquences des unions entre blancs et indiens, qui prennent exclusivement la forme de l'adultère et d'une « conduite peu glorieuse des enfants des maîtres ». Ces unions ne peuvent qu'aboutir à la " dégénération de la race maya » puis à son «extinction complète » (Pacheco Cruz, 1927). Parallèlement, pour l'élite yucatèque descendante de la « caste divine », le métissage n'est pas non plus souhaitable. Il est symbolisé par la « revanche » de Canek, leader d'une des premières rébellions indiennes d'importance (en 1761), mis en scène dans un roman écrit en 1940 par Ermilo Abreu Gómez. L’indien devient alors l'acteur principal de l'histoire et

18 Professeur, écrivain, historien, S. Pacheco Cruz a écrit de nombreux textes sur la culture et la société maya, notamment en tant que directeur du Département d'éducation de l'État de Yucatán, puis de l'État de Quintana Roo. 
la population blanche est renvoyée à la marge, dans une logique d'inversion de l'altérité et un nouveau constat d'échec du métissage.

Dans ce contexte, il me semble que le noir permet, au moins symboliquement, de rendre acceptable un métissage qui n'intègre que l'indien acculturé ou l'indien mort, un métissage imposé par le centre mais non souhaité par l'élite régionale, un métissage fondé sur l'instrumentalisation du monde maya afin de légitimer une "identité yucatèque " spécifique. Une telle « alchimie » se situe à deux niveaux, que je développerai dans cette dernière partie : d'une part, le noir rend nécessaire le métissage en symbolisant la menace du conflit racial, qui reste bien prégnante au lendemain de la Guerre des castes ; d'autre part, en étant appréhendé en termes raciaux, il inscrit par contraste le mestizo dans une logique de définition strictement ethnique, qui autorise à son tour l'élite à se réclamer du métissage en s'appropriant la culture et l'histoire indiennes.

Je reviendrai donc tout d'abord sur le premier point : loin d'en être absent, le noir est un acteur clé du métissage. Pour l'époque coloniale, Ben Vinson et Bob Vaughn considèrent que le noir a joué un rôle de passeur, d'intermédiaire entre populations blanches et indiennes, favorisant ainsi le processus de métissage au Mexique : « le métissage mexicain s'est fait, en grande partie, grâce aux afromexicains » (Vinson et Vaughn, 2004 : 15). Melchor Campos souligne lui aussi, dans le cas du Yucatán, l'importance des unions entre indiens ou mestizos et noirs ou mulâtres à la veille de l'indépendance (Campos Garcia, 2005 : 53). La proximité sociale entre castes inférieures a favorisé leur union et le métissage indien/blanc est de fait bien souvent passé par une étape intermédiaire indien/ mulâtre ou noir et mulâtre ou noir/blanc. Le personnage du negrito a-t-il également eu une fonction, au moins symboliquement, dans la politique du métissage de cette première moitié de XXe siècle ? Si la proximité sociale coloniale a rendu possible le métissage, en raison de la position d'entre-deux du noir, il me semble que la proximité sociale du XXe siècle agit davantage comme une double fascination-rejet, liée à l'ambiguïté du statut du noir entre quotidienneté et extériorité, entre popularité et marginalité sociale, entre appropriation et anormalité, entre intégration et racialisation. En ce sens, c'est parce qu'il incarne le risque d'une société non métissée que le noir est un des acteurs du métissage du début du XXe siècle.

Le negrito, mais aussi le chino ou le turco, symbolisent une altérité qui n'est pas intégrable dans le métissage, la menace d'une société où les différentes composantes de la population vivraient côte à côte, mais pas ensemble. Néanmoins le message porté par le negrito est encore plus immédiat car s'il est un « étranger » comme le chino ou le turco, on pourrait le qualifier d' « étranger de l'intérieur » tant il est, simultanément, proche. D'une part, du fait de son association à Cuba, le negrito incarne l'extériorité au même titre que le chino ou le turco. Mais, d'autre part, le Yucatán a bien connu historiquement la présence d'une population noire qui s'est intégrée aux structures sociales, notamment en ville. En 1742, pour le Yucatán, Gonzalo Aguirre Beltrán (1989 : 222) évalue la population indienne à 190032 personnes, la catégorie « afromestizo » représente le deuxième groupe de population (35 712), avant les « indomestizo » (19 588) et les « euromestizos » (17 660). Selon Jorge Ignacio Rubio Mañé (cité par Campos García, 2005 : 23), en 1789, la Province de Yucatán et Tabasco compte 72,8 \% d'indiens, 14,8 \% d'espagnols et mestizos et 12,4\% de noirs et mulâtres. De fait, on l'a vu, le personnage du noir est considéré par plusieurs 
auteurs comme un des «types populaires » ayant marqué Mérida au début du XXe siècle. Ce n'est pas le cas du chino, du turco, ni même de l'indien. Ce mélange de distance et de proximité, d'extériorité et de familiarité, de marginalisation et d'intégration, fait du negrito un personnage intermédiaire entre l'exotisme du chino ou du turco et la ressemblance du mestizo ou de la mestiza.

Un éditorial signé de Jorge Ignacio Rubio Mañe dans le Diario de Yucatán, paru le 21 décembre 1943, au moment même où le negrito Arredondo triomphe au théâtre Colonial, est significatif de ce rôle joué par le noir dans la société yucatèque du début du XXe siècle. Rubio Mañe, né à Mérida en 1904, a étudié aux États-Unis avant de travailler à l'Archivo General de la Nación et à l'Instituto Panamericano de Geografía e Historia à Mexico et d'être membre de l'Académie d'Histoire Mexicaine. Intellectuel reconnu, auteur de nombreux ouvrages et articles sur le Yucatán, il défend une position intermédiaire prônant une intégration de la péninsule à la nation, tout en valorisant une «identité yucatèque » spécifique. Il se situe ainsi au cœur de la réflexion sur le métissage et la nation. Dans cet article, il s'inquiète du « problème noir des noirs des EU » comme l'indique le titre de son texte. Ce « problème (doublement) noir » est lié au fait que les populations noires n'ont pas pu intégrer le modèle culturel européen : le souhait des noirs des ÉtatsUnis est de " paraître aussi cultivés intellectuellement que les descendants des anglais, des scandinaves et de tous les européens qui ont peuplé ce territoire. La seule chose qui les empêche de vivre avec les autres dans ce cadre, c'est la couleur de leur peau, qui les oblige à vivre isolés ». La différence avec le Mexique est alors bien rappelée : « ce n'est pas le problème du Mexique, où la culture espagnole est entrée en pleine compétition avec les cultures indiennes, et l'incorporation de l'indien à la civilisation a entraîné l'inverse : l'incorporation du blanc à la civilisation indienne ». Les États-Unis souhaitaient en outre « chasser » les noirs du pays et ont, au milieu du XIXe siècle, demandé « à une nation hispano-américaine la vente d'une portion de territoire pour y envoyer les noirs ». L'article parle du Yucatán, puis du Belize, du Costa Rica, du Guatemala. Cette inquiétude des États américains face à la population noire se prolonge, selon l'auteur, au XXe siècle et conduira ces États à évoquer un « retour» en Afrique. L'éditorial précise davantage encore l'objet de sa crainte illustrée par l'exemple des États-Unis : il redoute que, si « les deux races vivent continuellement de façon séparée, une conflagration sociale arrivera un jour ou l'autre, suite à laquelle l'une des deux races sera exterminée " et s'achève sur cette question : « deux races peuvent-elles vivre séparées dans le même pays ? ». Le noir apparaît ainsi comme un symbole de séparation, de division, de mise à l'écart associé au modèle étatsunien. Or dans le Yucatán, le souvenir de la Guerre des castes reste très présent et témoigne, pour les intellectuels de l'époque, des dangers d'une société officiellement ou, au moins, publiquement, ségréguée racialement ${ }^{19}$. La référence aux populations noires permet ainsi, à l'élite yucatèque, d'évoquer le pire des scénarios possibles (Guerre des castes, ségrégation et conflit raciaux) et rend plus acceptable la perspective du métissage avec les indiens.

19 Rappelons par exemple que le grand intellectuel yucatèque Justo Sierra O'Reilly lorsqu'il se rendit, en 1848, à Washington afin d'obtenir l'aide étatsunienne en échange de la souveraineté de la péninsule, qualifiait les mayas de « brutes infâmes qui se gavent de sang, d'incendies et de destruction. Je souhaite que cette race maudite disparaisse aujourd'hui et ne réapparaisse jamais entre nous » (Sierra O’Reilly, Suárez Navarro, 1993 : 56). 
Dès lors, la racialisation du noir contraste avec l'ethnicisation de l'indien, dont l'altérité devient ainsi plus facilement appropriable ou valorisable. De fait, et c'est le deuxième point, alors que le noir est, comme on l'a vu, construit dans une logique raciale, au moins au début du XXe siècle (blackface qui renvoie à la couleur de la peau, personnage social décrit en termes de pathologie), l'indien est, à la même époque, appréhendé en termes strictement culturels, qui insistent en particulier sur le vêtement et la langue (voir Redfield, 1946). L'altérité du negrito n'est pas celle de l'indien : c'est une altérité sans culture, sans civilisation, qui renvoie à un ailleurs exotique, lui-même non décrit. Le noir symbolise une « culture non qualifiée » qui « circule et survit comme les mauvaises herbes », la « communauté des bas-fonds » (Lhamon, 2008 : 104 et 105). Strictement appréhendé en termes raciaux, il n'a rien à " apporter » à la société yucatèque contrairement à l'indien dont la culture, la civilisation, l'ancestralité sont mises en avant en ce début de XXe siècle. Alors que le noir renvoie à une transgression identitaire à la fois recherchée et crainte (voir la fascination-répulsion pour les personnages de Francisco Montejo Baqueiro et Santiago Burgos Brito), le mestizo signifie, par contraste, une folklorisation et une normalisation de l'indien, processus qui facilitent son appropriation et son intégration dans le métissage.

Le métissage prend ainsi des configurations multiples : il permet de contrôler les dangers de la racialisation en associant celle-ci, de façon exclusive, au noir ; il permet d'intégrer l'indien à la nation en le convertissant en signes culturels appropriables. Le noir disparaît dans le métissage tout en l'ayant facilité, soit parce qu'il devient lui-même métis (époque coloniale) mais sans entrer dans la catégorie de mestizo réservée aux descendants d'indiens et européens, soit parce qu'il est le catalyseur de l'alchimie métisse dont il reste extérieur et même antinomique (première moitié du XXe siècle).

\section{Références bibliographiques}

AGUIRRE BELTRÁN Gonzalo (1989 [1946]) La población negra de México. Estudio etnohistórico, México, Universidad Veracruzana, Instituto Nacional Indigenista, Gobierno del Estado de Veracruz, Fondo de Cultura Económica.

ÁVILA DOMÍNGUEZ Freddy, PÉREZ MONTFORT Ricardo y RINAUDO Christian (Eds) (à paraître) Circulaciones culturales. Lo afrocaribeño entre Cartagena, Veracruz y La Habana, México D.F., CIESAS-IRD-Universidad de Cartagena-AFRODESC.

BASAVE BENÍTEZ Agustín (2002 [1992]) México mestizo. Análisis del nacionalismo mexicano en torno a la mestizofilia de Andrés Molina Enríquez, México, Fondo de Cultura Económica.

BÉTHUNE Christian (2007) Minstrelsy, L'Homme, (3) 183, pp. 147-161.

BOJORQUEZ URZAIZ Carlos (1986) Cubanos patriotas en Yucatán, Mérida, Fondo Editorial de la Facultad de Ciencias Antropológicas.

BOJORQUEZ URZAIZ Carlos (2000) La emigración cubana en Yucatán (1868-1898), México, Imagen Contemporáneo.

BRACAMONTE Y SOSA Pedro (2004) La Encarnacion de la Profecia Canek en Cisteil, México, CIESAS, Colección Peninsular.

BURGOS BRITO Santiago (1946) Tipos pintorescos de Yucatán, México, Editorial Cultura. 
CAMPOS GARCÍA Melchor (2005) Castas, Feligresía y Ciudadanía en Yucatán. Los afromestizos bajo en régimen constitucional español, 1750-1822, Mérida, CONACYT - Universidad Autónoma de Mérida.

CAREAGA VILIESID Lorena (2000) De llaves y cerrojos: Yucatán, Texas y Estados Unidos a mediados del siglo XIX, México, Instituto Mora.

CERVERA José Juan (2007) La gloria de la raza. Los chinos en Yucatán, Mérida, Instituto de Cultura de Yucatán.

CERVERA ANDRADE Alejandro (1947) El teatro regional de Yucatán, Mérida, Imp. Guerra.

COLE Catherine (1996) Reading blackface in Africa: Wonders taken for signs, Critical Inquiry, 23, pp. 183-215.

COLLÍ COLLÍ Mario Baltazar (2005) Componentes africanos en Quintana Roo, Mérida, UADY, Facultad de Ciencias Antropológicas.

CUEVAS SEBA María Teresa de Jesús y MAÑANA PLACENSIO Miguel Bolívar (1990) Los libaneses de Yucatán, Mérida, Impresiones Profesionales.

ECHÁNOVE TRUJILLO Carlos A. (1944) Enciclopedia Yucatanense. Tomo IV: Historia de la beneficencia, la educación, la medicina, etc. Conmemorativa del IV Centenario de Mérida y Valladolid (Yucatán), Ciudad de México, Edición oficial del Gobierno de Yucatán.

FELDMAN Heidi Carolyn (2009) Interrogating Blackface in the Afro-Peruvian Revival, Emisferica, (5) 2. URL: http://hemi.ps.tsoa.nyu.edu/hemi/es/e-misferica-52/feldman

FERNÁNDEZ REPETTO Francisco y NEGROE SIERRA Genny (1995) Una población perdida en la memoria: los negros de Yucatán, Mérida, Universidad Autónoma de Yucatán.

FLORESCANO Enrique (1997) Etnia, Estado y Nación. Ensayo sobre las identidades colectivas en México, México, Nuevo Siglo-Aguilar.

FLORESCANO Enrique (2002) Historia de las historias de la nación mexicana, México, Taurus.

FREDERIK L. A. (2001) The Contestation of Cuba's Public Sphere in National Theatre and the Transformation from Teatro Bufo to Teatro Nuevo, Gestos, 31, pp. 65-98.

GAMBOA GARIBALDI Arturo (1946) Historia del teatro y de la literatura dramática, in Carlos A. Echánove Trujillo Ed., Enciclopedia Yucatanense. Tomo V: Historia de la imprenta, el periodismo, el teatro, etc., Ciudad de México, Edición oficial del Gobierno de Yucatán.

HOFFMANN Odile (2006) Negros y afromestizos en México: viejas y nuevas lecturas de un mundo olvidado, Revista Mexicana de Sociologia, (68) 1, enero-marzo, pp. 103-135.

IRIGOYEN ROSADO Renan (1976) Esencia del Folklore de Yucatán, Mérida, Ediciones del Gobierno del Estado ( $2^{\mathrm{a}}$ edición).

KRAUZE Enrique (2007 [2005]) La presencia del pasado, México, Tusquets Editores.

LANE Jill (2005) Blackface Cuba, 1840-1895, Philadelphia, University of Pennsylvania Press.

LAPOINTE Marie (2006) Histoire du Yucatán. XIXe-XXIe siècles, Paris, L'Harmattan.

LEAL Rine (selección y prologo) (1975) Teatro bufo siglo XIX. Antología, tomo 1, La Habana, Biblioteca básica de literatura cubana, Editorial Arte y Literatura.

LEAL Rine (1980) Breve historia del Teatro Cubano, La Habana, Colección Panorama, Editorial Letras Cubanas.

LHAMON William T. (2008) Peaux blanches, masques noirs. Performances du blackface de Jim Crow à Michael Jackson, Paris, Kargo \& L'Éclat (édition originale : Raising Cain. Blackface performance from Jim Crow to Hip-Hop, Harvard University Press, 1998).

LOTT Eric (1993) Love and Theft: Blackface Minstrelsy and the American Working Class, New York and Oxford, Oxford University Press.

MALDONADO Roberto (1988) La influencia y participación de la música popular caribeña en la música popular de Yucatán, in Cultura del Caribe, Memoria del Festival Internacional de Cultura del Caribe, México, Unidad del Programa Cultural de las Fronteras/SEP, pp. 330-338.

MANZANILLA DORANTES Juan R. (1994) Teatro regional, relación entre Cuba y Yucatán, Revista de la Universidad Autónoma de Yucatán, 188, enero/febrero/marzo, pp. 78-87. 
MARTIATU INÉS María (prólogo y compilación) (2009) Bufo y nación. Interpelaciones desde el presente, La Habana, Editorial Letras Cubanas.

MONTEJO BAQUEIRO Francisco D. (1986 [1981]) Mérida en los años veinte, Mérida, Yucatán, México, Maldonado Editores, Colección historia y sociedad.

MONTENEGRO ROLON Orlando Enrique y DEL VALLE ARROYO Pablo Emilio (s.f.) El Danzón en México, Huellas, 67 y 68. URL: http://www.uninorte.edu.co/publicaciones/upload/ File/huellas_No67y68.pdf

MUÑOZ Fernando (1987) El teatro regional de Yucatán, México, Colección Escenología, Grupo Editorial Gaceta.

NOVELO Victoria (2009) Yucatecos en Cuba: etnografía de una migración, México, CIESAS, Publicaciones de la Casa Chata, Instituto de Cultura de Yucatán.

OROSA DÍAZ Jaime (1988) Historia de Yucatán, Mérida, Universidad Autónoma de Yucatán Ediciones.

PACHECO CRUZ Santiago (1927) El Cepo. Zarzuela histórica, Mérida, Yucatán.

PACHECO CRUZ Santiago (1928) La voz del amo. Zarzuela regional histórica, Mérida, Yucatán, Imprenta Oriente.

PÉREZ MONTFORT Ricardo (2007) De vaquerías, bombas, pichorradas y trova. Ecos del Caribe en la cultura popular yucateca 1890-1920, in Ricardo Pérez Montfort, Expresiones populares y estereotipos culturales en México. Siglos XIX y XX. Diez ensayos, México DF, CIESAS, Publicaciones de la Casa Chata, pp. 211-236.

PRIETO STAMBAUGH Antonio y ARMAND GARCÍA Oscar (Ed.) (2007) Ofelia Zapata «Petrona». Una vida dedicada al Teatro Regional, Mérida, Instituto de Cultura de Yucatán, ESAY-CINEY.

RÁMIREZ CANUL Marcos (2001) Música y músicos tradicionales de Quintana Roo, Chetumal, Instituto Quintanarroense de la Cultura-UQROO.

RAMÍREZ CARRILLO Luis Alfonso (2003) La Ronda de las Élites: Región y Poder en Yucatán, in Luis Alfonso Ramírez Carrillo Coord., Territorios, Actores y Poder. Regionalismos Emergentes en México, México, Universidad de Guadalajara, Centro Universitario de Ciencias Sociales y Humanidades, Universidad Autónoma de Yucatán, pp. 191-220.

RAMOS SMITH Maya (1991) El ballet en México en el siglo XIX. De la independencia al segundo imperio (1825-1867), México DF, Conaculta-Alianza Editorial.

REDFIELD Robert (1946) Raza y clases en Yucatán, in Carlos A. Echánove Trujillo Ed., Enciclopedia Yucatanense. Conmemorativa del IV Centenario de Mérida y Valladolid (Yucatán). Tomo VI: Yucatán actual, Ciudad de México, Edición oficial del Gobierno de Yucatán, (traduction de «Cooperation in Research», Carnegie Institution of Washington, 501, 1933).

REDONDO DOMÍNGUEZ Brígido (1994) Negritud en Campeche, Campeche, Ediciones del Congreso del Estado de Campeche.

Registro Yucateco (1845) Periódico literario, redactado por una sociedad de amigos. Tomo 1.

RESTALL Mathew (Ed.) (2005) Beyond Black and Red: African-Native Relations in Colonial Latin America, Albuquerque, University of New Mexico Press.

RIVERO Ramón and RIVERO Yeidy M. (2004) Caribbean Negritos, Blackface, and "Black" Voice in Puerto Rico, Television \& New Media, 5 (4), pp. 315-337.

SHRIMPTON MASSON Margaret (2002) Relecturas de la narrativa yucateca en el contexto del Caribe, Revista Mexicana del Caribe, 13, pp. 99-123.

SIERRA O'REILLY Justo y SUÁREZ NAVARRO Juan (testimonios) (1993) «Diario de nuestro viaje a los Estados Unidos». La Guerra de castas, México, Conaculta.

SUÁREZ DURÁN Esther (2008) El teatro bufo cubano, la vastedad de su universo, in Inés María Martiatu (prólogo y compilación) Bufo y nación, La Habana, Editorial Letras Cubanas, pp. 239-297.

TARACENA Arturo (2007) El Museo Yucateco y la reinvención de Yucatán. La prensa y la construcción del regionalismo peninsular, Península, 2 (1), primavera, pp. 13-46. 
TUYUB CASTILLO Gilma Rosaura (2005) El teatro regional yucateco, Mérida, Instituto de Cultura de Yucatán, Conaculta, Pacmyc.

VICTORIA OJEDA Jorge y CANTO ALCOCER Jorge (2006) San Fernando Aké. Microhistoria de una comunidad afroamericana en Yucatán, Mérida, Universidad Autónoma de Yucatán.

VINSON Ben y VAUGHN Bobby (2004) Afroméxico. El pulso de la población negra en México: una historia recordada, olvidada y vuelta a recordar, México, Fondo de Cultura Económica.

ZABALA Pilar, CUCINA Andrea, TIESLER Vera y NEFF Hector (2004) La población africana en la villa colonial de Campeche: un estudio interdisciplinario, in Los Investigadores de la Cultura Maya 12, tomo I, Campeche, Universidad Autónoma de Campeche, pp. 164-173. 


\title{
Negritos et mestizos à Mérida dans la première moitié du XXe siècle. Métissage, région, race

\author{
Elisabeth Cunin
}

\begin{abstract}
Dans la péninsule du Yucatán, au sud-est du Mexique, l'Indien incarne l'altérité, tant d'un point de vue scientifique que politique. Cet article se propose d'adopter un regard décalé en s'intéressant au " noir ": s'il est absent des travaux historiques locaux et des recherches ethnologiques, d'autres sources permettent néanmoins d'analyser le statut du « noir » dans le Yucatán, dans la première moitié du XXe siècle. Au-delà du récit dominant portant sur la confrontation entre l'Européen et l'Indien, l'ancrage caribéen du Yucatán déplace le débat sur l'«identité régionale » dans un cadre géographique et culturel plus large, renvoyant notamment au phénomène du blackface, d'accentuation de traits physiques noirs, dans une logique racisante. Ce blackface sera étudié au travers du personnage du negrito, et de la référence aux Negros. L'altérité différentielle entre Indiens et Noirs renvoie à des processus historiques et à des données démographiques, et révèle le regard porté par la société dominante sur ses minorités.
\end{abstract}

\section{Negritos and Mestizos in Merida at the Beginning of the 20th. Mestizaje, Region, Race \\ Elisabeth Cunin}

In the Yucatan peninsula, in Southeastern Mexico, the Indian represents otherness, both from a scientific and a political point of view. This article offers an original approach by focusing on black people, who do not appear in local historical works nor in ethnological research, but do appear in other sources, which enable us to analyze the status of "blackness" in Yucatan, at the beginning of the 20th century. Beyond the predominant tales on the confrontation between Europeans and Indians, the Caribbean dimension of Yucatan locates the debate on "regional identity" in a broader geographical and cultural frame. Within this framework, the phenomenon of "Blackface", i.e. the accentuation of black physical traits, in a racial logic, becomes relevant. I will base my analysis of "Blackface" on the figure of the negrito and on the reference to Negros. "Differential otherness" between Indians and Blacks is related to historical and demographic processes, and it reveals the vision the dominant society has on its minorities. 


\section{Negritos y mestizos en Mérida en la primera mitad del siglo XX. Mestizaje, región y raza \\ Elisabeth Cunin}

En la península de Yucatán, en el Sur-Este mexicano, «el indígena» encarna la alteridad, desde un punto de vista tanto científico como político. Este artículo propone adoptar una mirada original, interesándose por el «negro»: si bien es cierto que está ausente de los trabajos históricos locales y de las investigaciones etnológicas, otras fuentes, más cotidianas, más ordinarias, nos permiten analizar el estatus del «negro» en Yucatán, en la primera mitad del siglo XX. Más allá del relato dominante sobre la confrontación entre europeo e indígena, el anclaje caribeño de Yucatán ubica el debate sobre la «identidad regional» en un marco geográfico y cultural más amplio, en el cual encontramos en particular al fenómeno del blackface, es decir la acentuación de rasgos físicos negros, en una lógica racizante. Este blackface será estudiado a través del personaje del negrito, en el teatro regional yucateco, y de la referencia a los Negros, figuras populares de las calles de Mérida, a principio del siglo XX. La alteridad diferencial entre indígenas y negros remite a procesos históricos y datos demográficos, a la vez que es reveladora de la mirada de la sociedad dominante sobre sus minorías. 